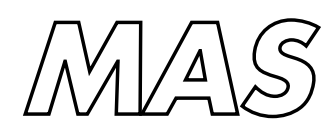

Modelling, Analysis and Simulation

\footnotetext{
Modelling, Analysis and Simulation cylinder functions
}

\author{
A. Gil, J. Segura, N.M. Temme \\ RePORT MAS-E0508 June 2005
}


$\mathrm{CWI}$ is the National Research Institute for Mathematics and Computer Science. It is sponsored by the Netherlands Organization for Scientific Research (NWO).

$\mathrm{CWI}$ is a founding member of ERCIM, the European Research Consortium for Informatics and Mathematics.

CWI's research has a theme-oriented structure and is grouped into four clusters. Listed below are the names of the clusters and in parentheses their acronyms.

Probability, Networks and Algorithms (PNA)

Software Engineering (SEN)

\section{Modelling, Analysis and Simulation (MAS)}

Information Systems (INS)

Copyright (C) 2005, Stichting Centrum voor Wiskunde en Informatica

P.O. Box 94079, 1090 GB Amsterdam (NL)

Kruislaan 413, 1098 SJ Amsterdam (NL)

Telephone +31205929333

Telefax +31205924199

ISSN 1386-3703 


\title{
Integral representations for computing real parabolic cylinder functions
}

\begin{abstract}
Integral representations are derived for the parabolic cylinder functions $U(a, x), V(a, x)$ and $W(a, x)$ and their derivatives. The new integrals will be used in numerical algorithms based on quadrature. They follow from contour integrals in the complex plane, by using methods from asymptotic analysis (saddle point and steepest descent methods), and are stable starting points for evaluating the functions $U(a, x), V(a, x)$ and $W(a, x)$ and their derivatives by quadrature rules. In particular, the new representations can be used for large parameter cases. Relations of the integral representations with uniform asymptotic expansions are also given. The algorithms will be given in a future paper.
\end{abstract}

2000 Mathematics Subject Classification: 33C15, 41A60, 65D20

Keywords and Phrases: parabolic cylinder functions, saddle point method, numerical evaluation of special functions, quadrature rules.

Note: This paper has been published in Numerische Mathematik 98, 105--134 (2004). 



\title{
Integral Representations for Computing Real Parabolic Cylinder Functions
}

\author{
Amparo Gil $^{1}$, Javier Segura ${ }^{2}$, Nico M. Temme ${ }^{3}$ \\ 1 Departamento de Matemáticas, U. Autónoma de Madrid, 28049-Madrid, Spain; \\ amparo.gil@uam.es \\ 2 Departamento de Matemáticas, Estadística y Computación, Univ. Cantabria, \\ 39005-Santander, Spain; javier.segura@unican.es \\ 3 CWI, P.O. Box 94079, 1090 GB Amsterdam, The Netherlands; nicot@cwi.nl
}

Received: date / Revised version: date

Summary Integral representations are derived for the parabolic cylinder functions $U(a, x), V(a, x)$ and $W(a, x)$ and their derivatives. The new integrals will be used in numerical algorithms based on quadrature. They follow from contour integrals in the complex plane, by using methods from asymptotic analysis (saddle point and steepest descent methods), and are stable starting points for evaluating the functions $U(a, x), V(a, x)$ and $W(a, x)$ and their derivatives by quadrature rules. In particular, the new representations can be used for large parameter cases. Relations of the integral representations with uniform asymptotic expansions are also given. The algorithms will be given in a future paper.

Mathematics Subject Classification (2000): 33C15, 41A60, 65D20. 


\section{Contents of the paper}

We give an overview of the structure of the paper.

Section 1 The basic properties of the parabolic cylinder functions $U(a, z)$ and $V(a, z)$ that are used in this paper.

Section 2 The integral representation of $U(a, x)$ for $a>0$.

2.1 the case $x \geq 0$.

2.2 the case $x \leq 0$.

2.3 a Wronskian relation for 4 integrals.

2.4 the relation with uniform asymptotic expansions.

Section 3 The integrals of $U(a, x)$ and $V(a, x)$ for $a<0$.

3.1 the case $-1 \leq t \leq 1$, where $t=x /(2 \sqrt{|a|})$.

3.1.1 a Wronskian relation for 4 integrals.

3.1.2 the relation with uniform asymptotic expansions.

3.2 the case $t \geq 1$.

3.2.1 a Wronskian relation for 4 integrals.

3.2.2 the relation with uniform asymptotic expansions.

3.3 the case $t \sim 1$.

3.4 the case $t \leq-1$.

Section 4 The $W$-function.

4.1 the standard solutions.

4.1.1 the function $\rho(a)$.

4.2 the case $a<0$.

4.3 the case $a>0$.

4.3.1 the case $t \geq 1$, where $t=x /(2 \sqrt{a})$.

4.3.2 the case $-1 \leq t \leq 1$.

4.3.3 unstable representations.

Section 5 Concluding remarks.

\section{Introduction}

The solutions of the differential equation

$$
\frac{d^{2} y}{d z^{2}}-\left(\frac{1}{4} z^{2}+a\right) y=0
$$

are called parabolic cylinder functions and are entire functions of $z$. As in [1], Chapter 19, [7], and [9] we denote two standard solutions of (1.1) by $U(a, z), V(a, z)$. Another notation found in the literature is $D_{\nu}(z)=U\left(-\nu-\frac{1}{2}, z\right)$. Special cases are Hermite polynomials, error functions and Fresnel integrals. 
Values at the origin are given by

$$
\begin{gathered}
U(a, 0)=\frac{\sqrt{\pi}}{2^{\frac{1}{2} a+\frac{1}{4}} \Gamma\left(\frac{3}{4}+\frac{1}{2} a\right)}, U^{\prime}(a, 0)=-\frac{\sqrt{\pi}}{2^{\frac{1}{2} a-\frac{1}{4}} \Gamma\left(\frac{1}{4}+\frac{1}{2} a\right)}, \\
V(a, 0)=\frac{\pi 2^{\frac{1}{2} a+\frac{1}{4}}}{\left[\Gamma\left(\frac{3}{4}-\frac{1}{2} a\right)\right]^{2} \Gamma\left(\frac{1}{4}+\frac{1}{2} a\right)}, V^{\prime}(a, 0)=\frac{\pi 2^{\frac{1}{2} a+\frac{3}{4}}}{\left[\Gamma\left(\frac{1}{4}-\frac{1}{2} a\right)\right]^{2} \Gamma\left(\frac{3}{4}+\frac{1}{2} a\right)} .
\end{gathered}
$$

Then we have

$$
\begin{aligned}
& U(a, z)=U(a, 0) y_{1}(a, z)+U^{\prime}(a, 0) y_{2}(a, z), \\
& V(a, z)=V(a, 0) y_{1}(a, z)+V^{\prime}(a, 0) y_{2}(a, z),
\end{aligned}
$$

where

$$
\begin{aligned}
y_{1}(a, z) & =e^{\frac{1}{4} z^{2}}{ }_{1} F_{1}\left(-\frac{1}{2} a+\frac{1}{4}, \frac{1}{2} ;-\frac{1}{2} z^{2}\right) \\
& =e^{-\frac{1}{4} z^{2}}{ }_{1} F_{1}\left(\frac{1}{2} a+\frac{1}{4}, \frac{1}{2} ; \frac{1}{2} z^{2}\right), \\
y_{2}(a, z) & =z e^{\frac{1}{4} z^{2}}{ }_{1} F_{1}\left(-\frac{1}{2} a+\frac{3}{4}, \frac{3}{2} ;-\frac{1}{2} z^{2}\right) \\
& =z e^{-\frac{1}{4} z^{2}}{ }_{1} F_{1}\left(\frac{1}{2} a+\frac{3}{4}, \frac{3}{2} ; \frac{1}{2} z^{2}\right),
\end{aligned}
$$

and the confluent hypergeometric function is defined by

$$
{ }_{1} F_{1}(a, c ; z)=\sum_{n=0}^{\infty} \frac{(a)_{n}}{(c)_{n}} \frac{z^{n}}{n !},
$$

with $(a)_{n}=\Gamma(a+n) / \Gamma(a), n=0,1,2, \ldots$

The functions $y_{1}(a, z)$ and $y_{2}(a, z)$ are the simplest even and odd solutions of (1.1) and the Wronskian of this pair is given by

$$
\mathcal{W}\left[y_{1}(z), y_{2}(z)\right]=y_{1}(z) y_{2}^{\prime}(z)-y_{1}^{\prime}(z) y_{2}(z)=1 .
$$

¿From a numerical point of view, the pair $\left\{y_{1}, y_{2}\right\}$ is not a satisfactory pair [4], because they have almost the same asymptotic behaviour at infinity,

The behaviour of $U(a, z)$ and $V(a, z)$ is, for large positive $z$ and $z \gg|a|$ :

$$
\begin{aligned}
& U(a, z)=e^{-\frac{1}{4} z^{2}} z^{-a-\frac{1}{2}}\left[1+\mathcal{O}\left(z^{-2}\right)\right], \\
& V(a, z)=\sqrt{2 / \pi} e^{\frac{1}{4} z^{2}} z^{a-\frac{1}{2}}\left[1+\mathcal{O}\left(z^{-2}\right)\right] .
\end{aligned}
$$

Clearly, numerical computations of $U(a, z)$ that are based on the representations in (1.3) and (1.4) should be done with great care, because of the loss of accuracy if $z$ becomes large. Also, for large $a$ these representations become useless. 
The Wronskian relation between $U(a, z)$ and $V(a, z)$ reads:

$$
\begin{gathered}
\mathcal{W}[U(a, z), V(a, z)]=\sqrt{2 / \pi} . \\
\mathcal{W}[U(a, z), U(a,-z)]=\frac{\sqrt{2 \pi}}{\Gamma\left(a+\frac{1}{2}\right)} .
\end{gathered}
$$

which shows that $U(a, z)$ and $V(a, z)$ are independent solutions of (1.1) for all values of $a$. Other relations are

$$
\begin{aligned}
& U(a, z)=\frac{\pi}{\cos ^{2} \pi a \Gamma\left(a+\frac{1}{2}\right)}[V(a,-z)-\sin \pi a V(a, z)], \\
& V(a, z)=\frac{\Gamma\left(a+\frac{1}{2}\right)}{\pi}[\sin \pi a U(a, z)+U(a,-z)] .
\end{aligned}
$$

Equation (1.1) has two turning points at $\pm 2 \sqrt{-a}$. For real parameters they become important if $a$ is negative, and the asymptotic behaviour of the solutions of (1.1) as $a \rightarrow-\infty$ changes significantly if $z$ crosses the turning points. At these points Airy functions are needed for describing the asymptotic behaviour.

The purpose of this paper is to give integral representations of $U(a, x)$ and $V(a, x)$ for real values of $a$ and $x$. We use integral representations from the literature and modify these by saddle point methods. In this way we obtain integrands that are non-oscillating, also for the case $a<0$. In particular, we can use the new representations for large parameter cases. In earlier papers [8] and [2] we have used these methods for obtaining stable integral representations for modified Bessel functions with pure imaginary order and for inhomogeneous Airy functions (Scorer functions).

We give relations of the integral representations with uniform asymptotic expansions, which are taken from [6] and [10]. We only give the expansions in terms of elementary functions. Uniform expansions in terms of Airy functions can be found in [6], and a modified form in [10].

We also consider solutions $W(a, \pm x)$ of the differential equation

$$
W^{\prime \prime}+\left(\frac{1}{4} x^{2}-a\right) W=0,
$$

a modified form of (1.1), again for real $a$ and $x$. Properties of $W(a, x)$ are given in $\S 4$, which can be found in [1] and [5].

In a future paper we give algorithms based on quadrature rules for evaluating the integral representations of $U(a, x), V(a, x)$ and $W(a, x)$. 
In [10] numerical and asymptotic aspects of the parabolic cylinder functions have been discussed, and we refer to this paper frequently. The notation of certain quantities is also as in [10]. The asymptotic methods referred to in this paper (saddle point methods) can be found in [7] and [11]. For an overview of the numerical aspects and software for the parabolic cylinder functions we refer to [3].

\section{Integral representations for $a>0$}

We derive integral representations for $U(a, x)$ and $U(a,-x)$. The computation of $V(a, x)$ for $a>0$ can be based on the second relation in (1.11). For $a>0$ the functions $U(a, x)$ and $U(a,-x)$ have a nonvanishing Wronskian relation (see (1.10)), and moreover, these functions constitute a numerically satisfactory pair of solutions of (1.1).

\subsection{The case $\boldsymbol{x} \geq \mathbf{0}$}

We take the integral (see[1], formula 19.5.4)

$$
U(a, x)=\frac{e^{\frac{1}{4} x^{2}}}{i \sqrt{2 \pi}} \int_{\mathcal{C}} e^{-x s+\frac{1}{2} s^{2}} s^{-a} \frac{d s}{\sqrt{s}},
$$

where $\mathcal{C}$ is a vertical line on which $\Re s>0$. On $\mathcal{C}$ we have $-\frac{1}{2} \pi<$ ph $s<\frac{1}{2} \pi$, and the many-valued function $s^{-a-1 / 2}$ assumes its principal value. The transformations

$$
x=2 t \sqrt{a}, \quad s=\sqrt{a} w
$$

give

$$
U(a, x)=\frac{e^{\frac{1}{4} x^{2}} a^{\frac{1}{4}-\frac{1}{2} a}}{i \sqrt{2 \pi}} \int_{\mathcal{C}} e^{a \phi(w)} \frac{d w}{\sqrt{w}}
$$

where

$$
\phi(w)=\frac{1}{2} w^{2}-2 t w-\ln w .
$$

The saddle points follow from solving

$$
\phi^{\prime}(w)=\frac{w^{2}-2 t w-1}{w}=0
$$

giving saddle points at $t \pm \sqrt{t^{2}+1}$. We take for the path $\mathcal{C}$ in $(2.3)$ the vertical through the positive saddle point

$$
w_{0}=t+\sqrt{t^{2}+1} .
$$


At this saddle point $\mathcal{C}$ coincides with the steepest descent path trough $w_{0}$. The complete steepest descent path follows from solving the equation $\Im[\phi(w)]=\Im\left[\phi\left(w_{0}\right)\right]$. In the present case $\Im\left[\phi\left(w_{0}\right)\right]=0$ and we obtain for the saddle point contour the equation

$$
\frac{1}{2} r^{2} \sin 2 \theta-2 \operatorname{tr} \sin \theta-\theta=0, \text { where } w=r e^{i \theta},
$$

which can be solved for $r=r(\theta)$ :

$$
r=\frac{t+\sqrt{t^{2}+\theta \cot \theta}}{\cos \theta}, \quad-\frac{1}{2} \pi<\theta<\frac{1}{2} \pi .
$$

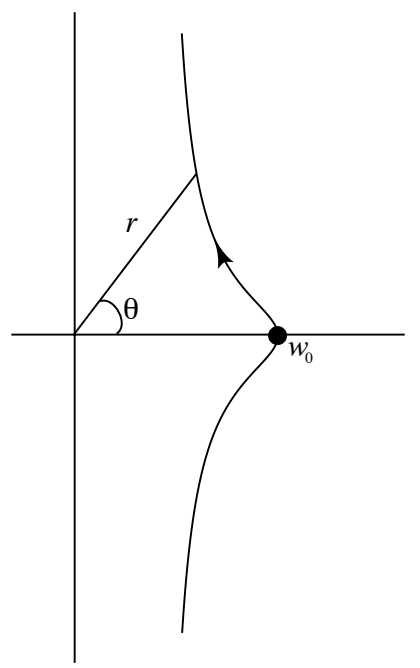

Figure 1. Steepest descent contour for the integral in (2.3).

Then (2.3) can be written as

$$
U(a, x)=\frac{e^{\frac{1}{4} x^{2}+a \phi\left(w_{0}\right)} a^{\frac{1}{4}-\frac{1}{2} a}}{\sqrt{2 \pi}} \int_{-\frac{1}{2} \pi}^{\frac{1}{2} \pi} e^{a \psi(\theta)} g(\theta) d \theta,
$$

where

$$
\psi(\theta)=\Re\left[\phi(w)-\phi\left(w_{0}\right)\right]=\frac{1}{2} r^{2} \cos 2 \theta-2 \operatorname{tr} \cos \theta-\ln r-\phi\left(w_{0}\right),(2.10)
$$

and

$$
\begin{aligned}
g(\theta) & =\Im\left[\frac{1}{\sqrt{w}} \frac{d w}{d \theta}\right]=\Im\left[e^{\frac{1}{2} i \theta} \frac{1}{\sqrt{r}}\left(\frac{d r}{d \theta}+i r\right)\right] \\
& =\frac{(2 \cos \theta+1) r^{2}-2 t r+1}{4 \sqrt{r} \cos \frac{1}{2} \theta \sqrt{t^{2}+\theta \cot \theta}} .
\end{aligned}
$$


The quantity $\tilde{\xi}$ defined by

$$
\widetilde{\xi}=\frac{1}{2}\left[t \sqrt{t^{2}+1}+\ln \left(t+\sqrt{t^{2}+1}\right)\right],
$$

is used in the asymptotic representation of $U(a, x)$ in this case; see [10], formula (2.29). We have

$$
\frac{1}{4} x^{2}+a \phi\left(w_{0}\right)=a\left[\frac{1}{2}-t \sqrt{t^{2}+1}-\ln \left(t+\sqrt{t^{2}+1}\right)\right]=a\left(\frac{1}{2}-2 \widetilde{\xi}\right) .
$$

This gives

$$
U(a, x)=\frac{a^{\frac{1}{4}} e^{-2 a \tilde{\xi}}}{\sqrt{2 \pi} \gamma(a)} \int_{-\frac{1}{2} \pi}^{\frac{1}{2} \pi} e^{a \psi(\theta)} g(\theta) d \theta
$$

where

$$
\gamma(a)=e^{-\frac{1}{2} a} a^{\frac{1}{2} a} .
$$

For the derivative $U^{\prime}(a, x)$ we can start from (2.1), and we have

$$
U^{\prime}(a, x)=\frac{e^{\frac{1}{4} x^{2}}}{i \sqrt{2 \pi}} \int_{\mathcal{C}} e^{-x s+\frac{1}{2} s^{2}} s^{-a}\left(\frac{1}{2} x-s\right) \frac{d s}{\sqrt{s}},
$$

This can be written as

$$
U^{\prime}(a, x)=-\frac{a^{\frac{3}{4}} e^{-2 a \widetilde{\xi}}}{\sqrt{2 \pi} \gamma(a)} \int_{-\frac{1}{2} \pi}^{\frac{1}{2} \pi} e^{a \psi(\theta)} h(\theta) d \theta
$$

where

$$
\begin{aligned}
h(\theta) & =-\Im\left[\frac{1}{\sqrt{w}} \frac{d w}{d \theta}(t-w)\right] \\
& =\frac{r^{3}-t r^{2}(2 \cos \theta-1)+r\left(2 t^{2}+1+2 \cos \theta\right)-t}{4 \sqrt{r} \cos \frac{1}{2} \theta \sqrt{t^{2}+\theta \cot \theta}} .
\end{aligned}
$$

\subsection{The case $\boldsymbol{x} \leq \mathbf{0}$}

This case can be done by using the representation of the previous section. However, when $t$ is a large negative number, the saddle point $w_{0}$ defined in (2.6) is close to origin, at which point the integrand of (2.3) is singular. As a consequence, the functions $\psi(\theta)$ and $g(\theta)$ in (2.9) have singularities close to the origin $\theta=0$ when $t$ is a large negative number. 
In the present case we start with the well-known integral representation (see [1], formula 19.5.3)

$$
U(a,-x)=\frac{e^{-\frac{1}{4} x^{2}}}{\Gamma\left(a+\frac{1}{2}\right)} \int_{0}^{\infty} s^{a-\frac{1}{2}} e^{-\frac{1}{2} s^{2}+x s} d s, \quad a>-\frac{1}{2} .
$$

There are no oscillations, but it is convenient to transform the integral in such a way that the saddle point is at the origin and a suitable normalization is obtained. The transformations (2.2) give

$$
U(a,-x)=\frac{a^{\frac{1}{2} a+\frac{1}{4}} e^{-\frac{1}{4} x^{2}}}{\Gamma\left(a+\frac{1}{2}\right)} \int_{0}^{\infty} e^{-a \phi(w)} \frac{d w}{\sqrt{w}},
$$

where $\phi(w)$ is given in (2.4). The positive saddle point $w_{0}$ is as in (2.6). We transform this point to the origin by writing $w=w_{0}(1+u)$, which gives

$$
U(a,-x)=\frac{a^{\frac{1}{4}} \sqrt{w_{0}} \gamma(a) e^{2 a \tilde{\xi}}}{\Gamma\left(a+\frac{1}{2}\right)} \int_{-1}^{\infty} e^{-a \psi(u)} \frac{d u}{\sqrt{1+u}},
$$

where we have used (2.13), $\gamma(a)$ is defined in (2.15), and

$$
\psi(u)=\frac{1}{2} w_{0}^{2} u^{2}+u-\ln (1+u) .
$$

For the derivative we have

$$
\begin{aligned}
U^{\prime}(a,-x)= & -\frac{a^{\frac{3}{4}} \sqrt{w_{0}} \gamma(a) e^{2 a \tilde{\xi}}}{\Gamma\left(a+\frac{1}{2}\right)} \\
& \times \int_{-1}^{\infty} e^{-a \psi(u)}\left(\sqrt{t^{2}+1}+w_{0} u\right) \frac{d u}{\sqrt{1+u}},
\end{aligned}
$$

To avoid numerical cancellation for small values of $u$ in the computation of $\psi(u)$ defined in (2.22), a specific code is needed for the evaluation of $\ln (1+u)-u$.

\subsection{A Wronskian for the integrals}

When checking the numerical algorithms the Wronskian relations in (1.9) and (1.10) can be used. When the parameters are large it is more convenient to use a Wronskian relation that is based on the integrals derived in the section. This gives a better control of the errors that occur in the quadrature rules, because large and small factors are not present in the integrals. 
Table 2.1. Values of $U(a, x)$ and $V(a, x)$ for several values of $a$, with $t=1.2$, $x=2 t \sqrt{a}$. We also give the Wronskian relation (1.9) (reduced to zero).

\begin{tabular}{rccr}
$a$ & $U(a, x)$ & $V(a, x)$ & Wronskian \\
\hline 10.1 & $.87742145116891(-016)$ & $.915940854687909(+015)$ & $.43(-17)$ \\
20.1 & $.28991030051243(-034)$ & $.196498319490114(+034)$ & $.40(-17)$ \\
30.1 & $.76172124886582(-054)$ & $.611136504670193(+053)$ & $.22(-15)$ \\
40.1 & $.37897794771218(-074)$ & $.106421744688740(+074)$ & $.61(-14)$ \\
50.1 & $.54336492182121(-095)$ & $.664057299719702(+094)$ & $.14(-16)$ \\
60.1 & $.28814488246502(-116)$ & $.114331948734753(+116)$ & $.14(-16)$ \\
70.1 & $.66706978761114(-138)$ & $.457283270654938(+137)$ & $.14(-16)$ \\
80.1 & $.75880403194555(-160)$ & $.376070790125305(+159)$ & $.22(-16)$ \\
90.1 & $.46343084237480(-182)$ & $.580588047014352(+181)$ & $.19(-16)$ \\
100.1 & $.16280901630040(-204)$ & $.156790547971731(+204)$ & $.19(-16)$ \\
\hline
\end{tabular}

We write (see (2.14), (2.17), (2.21), and (2.23), respectively)

$$
\begin{gathered}
U(a, x)=\frac{a^{\frac{1}{4}} e^{-2 a \tilde{\xi}}}{\sqrt{2 \pi} \gamma(a)} I(a, x), \\
U^{\prime}(a, x)=-\frac{a^{\frac{3}{4}} e^{-2 a \tilde{\xi}}}{\sqrt{2 \pi} \gamma(a)} I_{d}(a, x), \\
U(a,-x)=\frac{a^{\frac{1}{4}} \sqrt{w_{0}} \gamma(a) e^{2 a \tilde{\xi}}}{\Gamma\left(a+\frac{1}{2}\right)} J(a, x), \\
U^{\prime}(a,-x)=-\frac{a^{\frac{3}{4}} \sqrt{w_{0}} \gamma(a) e^{2 a \tilde{\xi}}}{\Gamma\left(a+\frac{1}{2}\right)} J_{d}(a, x) .
\end{gathered}
$$

Then the relation for the integrals reads

$$
I(a, x) J_{d}(a, x)+I_{d}(a, x) J(a, x)=\frac{2 \pi}{a \sqrt{w_{0}}} .
$$

In Table 2.1 we give values of $U(a, x)$ and $V(a, x)$ for several values of $a$, with $t=1.2, x=2 t \sqrt{a}$. The values of $I(a, x), I_{d}(a, x), J(a, x)$, $J_{d}(a, x)$ of $(2.24)-(2.27)$ are computed with double precision in one algorithm, with about 50 function evaluations for the trapezoidal rule for each value of $a$. We compared these values with those computed with the Maple 7.0 functions CylinderU $(a, x)$ and CylinderV $(a, x)$, with Digits $=20$, and found agreement for $a<40$. For the higher $a$-values, Maple's Digit parameter has to be increased. 


\subsection{Uniform asymptotic expansions for $\boldsymbol{a}>\mathbf{0}$}

The quantities related with the integrals are closely related with the uniform asymptotic expansions given in formulas (2.29), (2.33) and (2.34) of [10]. We have

$$
\begin{aligned}
& I(a, x)=\frac{\sqrt{\pi}}{\sqrt{a}\left(t^{2}+1\right)^{1 / 4}} \widetilde{F}_{\mu}(t), \quad I_{d}(a, x)=\frac{\sqrt{\pi}\left(t^{2}+1\right)^{1 / 4}}{\sqrt{a}} \widetilde{G}_{\mu}(t), \\
& J(a, x)=\frac{\sqrt{\pi}}{\sqrt{a w_{0}}\left(t^{2}+1\right)^{1 / 4}} \widetilde{P}_{\mu}(t), J_{d}(a, x)=\frac{\sqrt{\pi}\left(t^{2}+1\right)^{1 / 4}}{\sqrt{a w_{0}}} \widetilde{Q}_{\mu}(t),
\end{aligned}
$$

where $\widetilde{F}_{\mu}(t), \widetilde{G}_{\mu}(t), \widetilde{P}_{\mu}(t)$, and $\widetilde{Q}_{\mu}(t)$ are supplied with asymptotic expansions that have a double asymptotic property: one of the parameters $a$ or $t$ (or both) should be large. Recurrence relations for the coefficients of the expansions are given in [10].

\section{Integral representations for $a<0$}

We give integral representations for $U(-a, x)$ and $V(-a, x)$, with $a>0$, and we consider three $x$-intervals. Let $t=x /(2 \sqrt{a})$. The differential equation (1.1) becomes for $U(-a, 2 t \sqrt{a})$ and $V(-a, 2 t \sqrt{a})$ in terms of $t$

$$
\frac{d^{2} y}{d t^{2}}-4 a^{2}\left(t^{2}-1\right) y=0
$$

which has turning points at $t= \pm 1$. Consequently, we consider the intervals $t \leq-1,|t| \leq 1$ and $t \geq 1$. We start with the middle interval, where the oscillations occur.

\subsection{The case $-1 \leq t \leq 1$}

We consider the integral

$$
Y(a, x)=\int_{0}^{\infty} e^{-\frac{1}{2} s^{2}+x i s} s^{a-\frac{1}{2}} d s, \quad \Re a>-\frac{1}{2} .
$$

Using (2.19), we see that

$$
Y(a, x)=\Gamma\left(a+\frac{1}{2}\right) e^{-\frac{1}{4} x^{2}} U(a,-i x) .
$$

We also have

$$
\sqrt{\frac{2}{\pi}} e^{-\frac{1}{2} \pi i a+\frac{1}{4} \pi i} U(a,-i x)=U(-a, x) / \Gamma\left(a+\frac{1}{2}\right)+i V(-a, x) .
$$


This follows from using the initial values in (1.2) and those of $Y(a, x)$. It also follows from the relations in (1.11) and 19.4.6 in [1].

Hence,

$$
\sqrt{\frac{2}{\pi}} e^{-\frac{1}{2} \pi i a+\frac{1}{4} \pi i} e^{\frac{1}{4} x^{2}} Y(a, x)=U(-a, x)+i \Gamma\left(a+\frac{1}{2}\right) V(-a, x) .
$$

We see that the single integral (3.2) produces $U(-a, x)$ and $V(-a, x)$ by taking real and imaginary parts.

We proceed with $Y(a, x)$, and the transformations $x=2 \sqrt{a} t, s=$ $\sqrt{a} w$ give

$$
Y(a, x)=a^{a / 2+1 / 4} \int_{0}^{\infty} e^{-a \phi(w)} \frac{d w}{\sqrt{w}}
$$

where

$$
\phi(w)=\frac{1}{2} w^{2}-2 i t w-\ln w .
$$

We consider a path through the saddle point

$$
w_{+}=i t+\sqrt{1-t^{2}} \text {. }
$$

We have

$$
\phi\left(w_{+}\right)=\frac{1}{2}+t^{2}+2 i\left(\eta-\frac{1}{4} \pi\right), \quad \eta=\frac{1}{2}\left(\arccos t-t \sqrt{1-t^{2}}\right),
$$

where arccost has values in $[0, \pi]$ for $t \in[-1,1]$.

The path of steepest descent starts at $w=0$, runs through $w_{+}$, and terminates at $+\infty$; see Figure 2. The path follows from solving the equation

$$
\Im \phi(w)=\Im \phi\left(w_{+}\right),
$$

that is, from solving

$$
\frac{1}{2} r^{2} \sin 2 \theta-2 t r \cos \theta-\theta-2 \eta+\frac{1}{2} \pi=0,
$$

where $w=r e^{i \theta}$. The solution of (3.11) reads

$$
\begin{aligned}
& r=\frac{t \cos \theta+\sigma \sqrt{t^{2} \cos ^{2} \theta+\sin \theta \cos \theta\left(\theta+2 \eta-\frac{1}{2} \pi\right)}}{\sin \theta \cos \theta}, \\
& 0 \leq \theta \leq \theta_{0},
\end{aligned}
$$

where $\theta_{0}=-2 \eta+\frac{1}{2} \pi$; the square root is non-negative. The number $\sigma$ equals -1 when ph $w_{+} \leq \theta \leq \theta_{0}$, and +1 when $0 \leq \theta \leq \operatorname{ph} w_{+}$. Observe that ph $w_{+}=\frac{1}{2} \pi-\arccos t$. When $\theta=\theta_{0}$, we have $r=0$; when $\theta=$ ph $w_{+}$, we have $r=1$, and $\theta=0$ gives $r=\infty$. For $t=0$ the path coincides with the positive real axis. When $t<0$ the paths are in the lower half plane, and follow from those for $t>0$ by symmetry. 


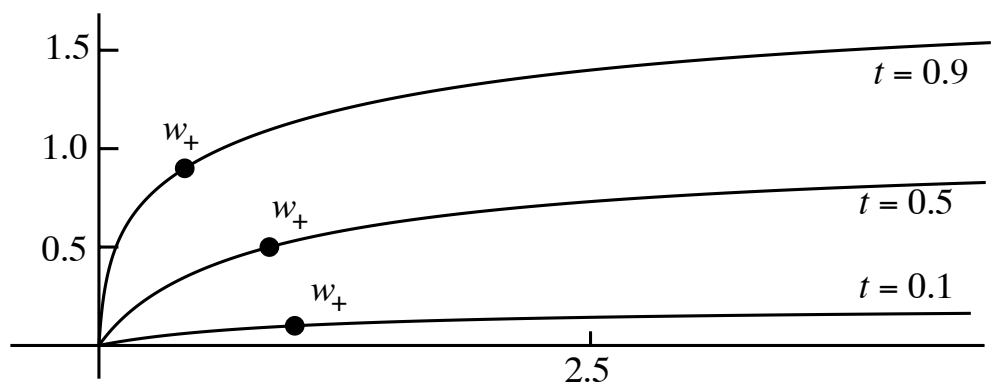

Figure 2. Steepest descent contours for the integral in (3.6) for $t=0.1,0.5,0.9$.

A simple approximation of the path is given by (we write $w=$ $u+i v)$

$$
v=\frac{u t\left(1+u_{+}\right)}{u+u_{+}^{2}}, \quad u_{+}=\sqrt{1-t^{2}} .
$$

This path runs through the point $w_{+}=u_{+}+i t$, and has the same slope at this point as the exact steepest descent path, that is, $d v / d u=$ $t /\left(1+u_{+}\right)$for $u=u_{+}$.

For $t=1$ the steepest descent path runs from the origin to $w_{+}=i$ along the imaginary axis, and from $i$ to $2 i+\infty$. For more details on the case $t \geq 1$ we refer to $\S 3.2$.

Integrating (3.6) with respect to $\theta$ we obtain

$$
\begin{aligned}
U(-a, x)+ & i \Gamma\left(a+\frac{1}{2}\right) V(-a, x)= \\
= & \sqrt{\frac{2}{\pi}} a^{\frac{1}{4}} \gamma(a) e^{-i\left(2 a \eta-\frac{1}{4} \pi\right)} \int_{0}^{\theta_{0}} e^{-a \psi(\theta)} g(\theta) d \theta,
\end{aligned}
$$

where $\gamma(a)$ is defined in (2.15),

$$
\psi(\theta)=\frac{1}{2} r^{2} \cos 2 \theta+2 t r \sin \theta-\ln r-t^{2}-\frac{1}{2},
$$

and

$$
g(\theta)=-\frac{e^{i \theta / 2}}{\sqrt{r}}\left(\frac{d r}{d \theta}+i r\right) .
$$

We write the representations for $U(-a, x)$ and $V(-a, x)$ in real form, with trigonometric functions that correspond with those in [6] and $[10]$. We first write

$$
g(\theta)=g_{1}(\theta)-i g_{2}(\theta),
$$

where $g_{j}(\theta), j=1,2$, are real. That is, by (3.16),

$$
g_{1}(\theta)=-\frac{d r}{d \theta} \frac{\cos \left(\frac{1}{2} \theta\right)}{\sqrt{r}}+r \frac{\sin \left(\frac{1}{2} \theta\right)}{\sqrt{r}},
$$




$$
g_{2}(\theta)=r \frac{\cos \left(\frac{1}{2} \theta\right)}{\sqrt{r}}+\frac{d r}{d \theta} \frac{\sin \left(\frac{1}{2} \theta\right)}{\sqrt{r}} .
$$

Then we have

$$
\begin{gathered}
U(-a, x)=\sqrt{\frac{2}{\pi}} a^{\frac{1}{4}} \gamma(a)\left[(\sin \lambda) G_{1}+(\cos \lambda) G_{2}\right], \\
V(-a, x)=\sqrt{\frac{2}{\pi}} a^{\frac{1}{4}} \frac{\gamma(a)}{\Gamma\left(a+\frac{1}{2}\right)}\left[(\cos \lambda) G_{1}-(\sin \lambda) G_{2}\right],
\end{gathered}
$$

where

$$
\lambda=2 a \eta+\frac{1}{4} \pi
$$

and

$$
G_{j}=\int_{0}^{\theta_{0}} e^{-a \psi(\theta)} g_{j}(\theta) d \theta, \quad j=1,2 .
$$

For the derivatives we find, using (3.2) and (3.5),

$$
\begin{aligned}
& U^{\prime}(-a, x)+i \Gamma\left(a+\frac{1}{2}\right) V^{\prime}(-a, x)= \\
& \quad \sqrt{\frac{2}{\pi}} a^{\frac{3}{4}} \gamma(a) e^{-i\left(2 a \eta-\frac{1}{4} \pi\right)} \int_{0}^{\theta_{0}} e^{-a \psi(\theta)} h(\theta) d \theta .
\end{aligned}
$$

where

$$
h(\theta)=(t+i w) g(\theta)=h_{1}(\theta)-i h_{2}(\theta) .
$$

That is, by (3.25), (3.18) and (3.19),

$$
\begin{aligned}
& h_{1}(\theta)=(t-r \sin \theta) g_{1}(\theta)+r \cos \theta g_{2}(\theta), \\
& h_{2}(\theta)=r \cos \theta g_{1}(\theta)-(t-r \sin \theta) g_{2}(\theta) .
\end{aligned}
$$

Then we have

$$
\begin{gathered}
U^{\prime}(-a, x)=\sqrt{\frac{2}{\pi}} a^{\frac{3}{4}} \gamma(a)\left[(\sin \lambda) H_{1}+(\cos \lambda) H_{2}\right], \\
V^{\prime}(-a, x)=\sqrt{\frac{2}{\pi}} \frac{a^{\frac{3}{4}} \gamma(a)}{\Gamma\left(a+\frac{1}{2}\right)}\left[(\cos \lambda) H_{1}-(\sin \lambda) H_{2}\right] .
\end{gathered}
$$

where $\lambda$ is given in Eq. (3.22) and

$$
H_{j}=\int_{0}^{\pi} e^{-a \psi(\theta)} h_{j}(\theta) d \theta, \quad j=1,2 .
$$


3.1.1 A Wronskian for the integrals By using (1.9) and the integrals in $(3.23)$ and $(3.30)$, we find the relation

$$
H_{1} G_{2}-G_{1} H_{2}=\sqrt{\frac{\pi}{2}} \frac{\Gamma\left(a+\frac{1}{2}\right)}{a \gamma^{2}(a)}=\frac{\pi}{a} \Gamma^{*}\left(a+\frac{1}{2}\right),
$$

where

$$
\Gamma\left(a+\frac{1}{2}\right)=\sqrt{2 \pi} \gamma^{2}(a) \Gamma^{*}\left(a+\frac{1}{2}\right), \quad \Gamma^{*}\left(a+\frac{1}{2}\right)=1+\mathcal{O}(1 / a),
$$

as $a \rightarrow \infty$. Hence, for large $a$, the right-hand side in (3.31) is of order $\frac{\pi}{a}[1+\mathcal{O}(1 / a)]$ (see also formula (3.28) in $\left.[10]\right)$. The relation in (3.31) can be used for testing the numerical algorithms.

3.1.2 Uniform asymptotic expansions $-1<\boldsymbol{t}<1$ The relationship of the integrals $G_{j}, H_{j}$ with uniform expansions follows from (2.23), (2.24) and (2.27) of [10]. These expansions are the same as in [6]. On the other hand, we can derive modified expansions (a main topic in [10]), by using (2.29) and (2.33) of that reference. From (3.4) and by changing $t$ to $-i t$ in(2.29) of [10], we obtain

$$
\begin{gathered}
U(-a, x)+i \Gamma\left(a+\frac{1}{2}\right) V(-a, x)= \\
\Gamma^{*}\left(a+\frac{1}{2}\right) \frac{\sqrt{2} \gamma(a) e^{\frac{1}{4} \pi i-2 i a \eta}}{a^{\frac{1}{4}}\left(1-t^{2}\right)^{\frac{1}{4}}} \widetilde{F}_{\mu}(-i t), \\
U^{\prime}(-a, x)+i \Gamma\left(a+\frac{1}{2}\right) V^{\prime}(-a, x)= \\
-\Gamma^{*}\left(a+\frac{1}{2}\right) \sqrt{2} a^{\frac{1}{4}} \gamma(a) e^{-\frac{1}{4} \pi i-2 i a \eta}\left(1-t^{2}\right)^{\frac{1}{4}} \widetilde{G}_{\mu}(-i t),
\end{gathered}
$$

where $\widetilde{F}_{\mu}(-i t)$ and $\widetilde{G}_{\mu}(-i t)$ have the asymptotic expansions

$$
\widetilde{F}_{\mu}(-i t) \sim \sum_{s=0}^{\infty}(-1)^{s} \frac{\phi_{s}\left(\tau^{*}\right)}{(2 a)^{s}}, \quad \widetilde{G}_{\mu}(-i t) \sim \sum_{s=0}^{\infty}(-1)^{s} \frac{\psi_{s}\left(\tau^{*}\right)}{(2 a)^{s}}
$$

as $a \rightarrow \infty$, uniformly for $t \in[-1+\delta, 1-\delta]$. The quantity $\tau^{*}$ is defined by

$$
\tau^{*}=-\frac{1}{2}\left(\frac{i t}{\sqrt{1-t^{2}}}+1\right) \text {. }
$$

The polynomials $\phi_{s}$ and $\psi_{s}$ are given in (2.11) and (2.16) of [10], with recursion relations. The first fraction at the right-hand sides of (3.33) and (3.34) has the asymptotic estimate $1+\mathcal{O}(1 / a)$ (see also formula (3.28) in $[10])$. 


\subsection{The case $\boldsymbol{t} \geq 1$}

We use the the integral for $Y(a, x)$ given in (3.6) with $\phi(w)$ given in (3.7). The saddle points are now purely imaginary:

$$
w_{-}=i t-i \sqrt{t^{2}-1}, \quad w_{+}=i t+i \sqrt{t^{2}-1} .
$$

We have

$$
\begin{aligned}
& \phi\left(w_{ \pm}\right)=\frac{1}{2}+t^{2}-\frac{1}{2} \pi i \pm 2 \xi, \\
& \xi=\frac{1}{2}\left[t \sqrt{t^{2}-1}-\ln \left(t+\sqrt{t^{2}-1}\right)\right] .
\end{aligned}
$$

The quantity $\xi$ is also is used in the asymptotic representation of $U(-a, x)$ for this case; see [6] and [10].

The path of steepest descent starts at $w=0$, runs through $w_{-}$ and $w_{+}$on the positive imaginary axis, and from $w_{+}$to $+\infty$. The path from $w_{+}$to $+\infty$ follows from solving the equation

$$
\Im \phi(w)=-\frac{1}{2} \pi,
$$

that is, from solving

$$
\frac{1}{2} r^{2} \sin 2 \theta-2 t r \cos \theta-\theta+\frac{1}{2} \pi=0,
$$

where $w=r e^{i \theta}$. The solution of (3.40) reads

$$
r=\frac{t \cos \theta+\sqrt{t^{2} \cos ^{2} \theta+\sin \theta \cos \theta\left(\theta-\frac{1}{2} \pi\right)}}{\sin \theta \cos \theta}, \quad 0 \leq \theta \leq \frac{1}{2} \pi .
$$

The square root is positive, unless when $t=1$ and $\theta=\frac{1}{2} \pi$.

We obtain

$$
\begin{gathered}
U(-a, x)+i \Gamma(a+1 / 2) V(-a, x)=\sqrt{\frac{2}{\pi}} a^{\frac{1}{4}} \gamma(a)\left[e^{-2 a \xi+\frac{1}{4} \pi i}\right. \\
\left.\times \int_{0}^{\frac{1}{2} \pi} e^{-a \psi(\theta)} g(\theta) d \theta+i e^{2 a \xi} \int_{0}^{r+} e^{a \widetilde{\phi}(v)} \frac{d v}{\sqrt{v}}\right]
\end{gathered}
$$

where $\widetilde{\phi}(v)=\phi\left(w_{-}\right)-\phi(i v), g(\theta)$ as in (3.16), and $\psi(\theta)=\phi(w)-$ $\phi\left(w_{+}\right)=\Re\left[\phi(w)-\phi\left(w_{+}\right)\right], w=r e^{i \theta}$, now with $r$ defined in (3.41). Explicitly,

$$
\begin{aligned}
& \widetilde{\phi}(v)=\frac{1}{2} v^{2}-2 t v+\ln v-\frac{1}{2} r_{-}^{2}+2 t r_{-}-\ln r_{-}, \\
& \psi(\theta)=\frac{1}{2} r^{2} \cos 2 \theta+2 t r \sin \theta-\ln r-\frac{1}{2}-t^{2}-2 \xi .
\end{aligned}
$$


where

$$
r_{-}=t-\sqrt{t^{2}-1}
$$

Considering the real and imaginary parts on both sides of (3.42), we see that for $V(-a, x)$ we need the $v$-integral with the dominant factor $e^{2 a \xi}$ and part of the $\theta$-integral. When $t \sim 1$ (in fact, when $a \xi$ is small) both integrals are of the same asymptotic importance. The dominant saddle point in the $v$-integral is $r_{-}$; in the $\theta$-integral the dominant point is the upper limit.

When we write

$$
e^{\frac{1}{4} \pi i} g(\theta)=g_{1}(\theta)+i g_{2}(\theta),
$$

where $g_{1}(\theta)$ and $g_{2}(\theta)$ are real, we have

and

$$
U(-a, x)=\sqrt{\frac{2}{\pi}} a^{\frac{1}{4}} \gamma(a) e^{-2 a \xi} G_{1}
$$

$$
V(-a, x)=\sqrt{\frac{2}{\pi}} a^{\frac{1}{4}} \frac{\gamma(a) e^{2 a \xi}}{\Gamma\left(a+\frac{1}{2}\right)}\left(e^{-4 a \xi} G_{2}+G_{3}\right) .
$$

where (for $j=1,2$ )

$$
G_{j}=\int_{0}^{\frac{1}{2} \pi} e^{-a \psi(\theta)} g_{j}(\theta) d \theta, \quad G_{3}=\int_{0}^{r_{+}} e^{a \widetilde{\phi}(v)} \frac{d v}{\sqrt{v}} .
$$

For the derivatives we have

$$
\begin{array}{r}
U^{\prime}(-a, x)+i \Gamma\left(a+\frac{1}{2}\right) V^{\prime}(-a, x)=\sqrt{\frac{2}{\pi}} a^{\frac{3}{4}} \gamma(a)\left[e^{-2 a \xi+\frac{1}{4} \pi i}\right. \\
\left.\times \int_{0}^{\frac{1}{2} \pi} e^{-a \psi(\theta)} h(\theta) d \theta+i e^{2 a \xi} \int_{0}^{r_{+}} e^{a \widetilde{\phi}(v)}(t-v) \frac{d v}{\sqrt{v}}\right],
\end{array}
$$

where $h(\theta)=(t+i w) g(\theta)$. When we write

$$
e^{\frac{1}{4} \pi i} h(\theta)=h_{1}(\theta)+i h_{2}(\theta),
$$

where $h_{1}(\theta)$ and $h_{2}(\theta)$ are real, we have

$$
U^{\prime}(-a, x)=\sqrt{\frac{2}{\pi}} a^{\frac{3}{4}} \gamma(a) e^{-2 a \xi} H_{1},
$$

and

$$
V^{\prime}(-a, x)=\sqrt{\frac{2}{\pi}} a^{\frac{3}{4}} \frac{\gamma(a) e^{2 a \xi}}{\Gamma\left(a+\frac{1}{2}\right)}\left[e^{-4 a \xi} H_{2}+H_{3}\right] .
$$

where (for $j=1,2$ )

$$
H_{j}=\int_{0}^{\frac{1}{2} \pi} e^{-a \psi(\theta)} h_{j}(\theta) d \theta, \quad H_{3}=\int_{0}^{r_{+}} e^{a \widetilde{\phi}(v)}(t-v) \frac{d v}{\sqrt{v}} .
$$


3.2.1 A Wronskian for the integrals By using the Wronskian relation in (1.9) and the integrals in (3.48) and (3.53), we obtain (cf. (3.31))

$$
\begin{gathered}
e^{-4 a \xi}\left(G_{1} H_{2}-H_{1} G_{2}\right)+\left(G_{1} H_{3}-H_{1} G_{3}\right) \\
=\sqrt{\frac{\pi}{2}} \frac{\Gamma\left(a+\frac{1}{2}\right)}{a \gamma^{2}(a)}=\frac{\pi}{a} \Gamma^{*}\left(a+\frac{1}{2}\right) .
\end{gathered}
$$

The relation in (3.54) can be used for testing the numerical algorithms.

3.2.2 Uniform asymptotic expansions for $\boldsymbol{t}>1$ We give the relationship of the integrals with the uniform expansions given in (2.9), (2.14), (2.18) and (2.29) of [10]. We have

$$
\begin{aligned}
& G_{1}=\frac{\sqrt{\pi}}{2 \sqrt{a}\left(t^{2}-1\right)^{\frac{1}{4}}} F_{\mu}(t), \\
& H_{1}=-\frac{\sqrt{\pi}\left(t^{2}-1\right)^{\frac{1}{4}}}{2 \sqrt{a}} G_{\mu}(t), \\
& e^{-4 a \xi} G_{2}+G_{3}=\frac{\Gamma\left(a+\frac{1}{2}\right) e^{a} a^{-a-\frac{1}{2}}}{\sqrt{2}\left(t^{2}-1\right)^{\frac{1}{4}}} P_{\mu}(t), \\
& e^{-4 a \xi} H_{2}+H_{3}=\frac{\Gamma\left(a+\frac{1}{2}\right) e^{a} a^{-a-\frac{1}{2}}\left(t^{2}-1\right)^{\frac{1}{4}}}{\sqrt{2}} Q_{\mu}(t),
\end{aligned}
$$

where $F_{\mu}(t), G_{\mu}(t), P_{\mu}(t)$, and $Q_{\mu}(t)$ are supplied with asymptotic expansions that have a double asymptotic property: one of the parameters $a$ or $t$ (or both) should be large; $t \geq 1+\delta$. Recurrence relations for the coefficients of the expansions are given in [10].

\subsection{The case $\boldsymbol{t} \sim 1$}

For $t \sim 1$ the contours used in $\S 3.1$ becomes less suitable for numerical quadrature. For example, we see in Figure 2 that the saddle point $w_{+}$approaches the imaginary unit when $t \uparrow 1$, and that the path becomes non-smooth when $t=1$. For numerical calculations we may consider uniform Airy-type asymptotic expansions if $t \sim 1$, and we will investigate later if this is indeed the best approach. But we also investigate if a modified contour can be used for numerical quadrature. 
We use for $t \sim 1$ the representation of $Y(a, x)$ in (3.6). We write $w=u+i v$, and integrate with respect to $v$ along the line segment from the origin to $w_{+}$, and then along the horizontal path from $w_{+}$ to $w_{+}+\infty$ with respect to $u$. In the first integral we substitute $v=$ $t(1-p)$, and integrate with respect to $p$. Observe that for $t \geq 1$ the point $w_{+}$is on the imaginary axis, and for this case no difficulties arise when $t \sim 1$, because the path is already split up into two parts; see $§ 3.2$.

It is not difficult to verify that the representations in (3.20), (3.21), (3.28) and (3.29) can be obtained, with $G_{j}, H_{j}$ replaced with $\widetilde{G}_{j}, \widetilde{H}_{j}$ $(j=1,2)$, where

$$
\begin{aligned}
& \widetilde{G}_{j}=\int_{0}^{1} e^{-a \psi_{r}^{(1)}(p)} g_{j}^{(1)}(p) d p \\
&+\int_{0}^{\infty} \frac{e^{-a \psi_{r}^{(2)}(u)}}{\left(1+2 u \sqrt{\left(1-t^{2}\right.}+u^{2}\right)^{\frac{1}{4}}} g_{j}^{(2)}(u) d u, \\
& \widetilde{H}_{j}=\int_{0}^{1} e^{-a \psi_{r}^{(1)}(p)} h_{j}^{(1)}(p) d p \\
&+\int_{0}^{\infty} \frac{e^{-a \psi_{r}^{(2)}(u)}}{\left(1+2 u \sqrt{\left(1-t^{2}\right.}+u^{2}\right)^{\frac{1}{4}}} h_{j}^{(2)}(u) d u
\end{aligned}
$$

where

$$
\begin{aligned}
& \psi_{r}^{(1)}(p)=\frac{1}{2} p^{2}\left(1-2 t^{2}\right)-p-\ln (1-p) \\
& \psi_{r}^{(2)}(u)=\frac{1}{2} u+u \sqrt{1-t^{2}}-\frac{1}{2} \ln \left(1+2 u \sqrt{\left(1-t^{2}\right.}+u^{2}\right) \\
& \psi_{i}^{(1)}(p)=p^{2} t \sqrt{1-t^{2}}, \\
& \psi_{i}^{(2)}(u)=\arctan \frac{u t}{1+u \sqrt{1-t^{2}}}-t u \\
& g_{1}^{(1)}(p)-i g_{2}^{(1)}(p)=e^{\frac{1}{2} i \tau-a i \psi_{i}^{(1)}(p)}, \quad \tau=\arcsin t \\
& g_{1}^{(2)}(u)-i g_{2}^{(2)}(u)=e^{-\frac{1}{2} i \tau-a i \psi_{i}^{(2)}(u)+i \arctan \frac{u t}{1+u \sqrt{1-t^{2}}}}
\end{aligned}
$$




$$
\begin{aligned}
& h_{1}^{(1)}(p)=t p g_{1}^{(1)}(p)+\sqrt{1-t^{2}}(1-p) g_{2}^{(1)}(p), \\
& h_{2}^{(1)}(p)=t p g_{2}^{(1)}(p)-\sqrt{1-t^{2}}(1-p) g_{1}^{(1)}(p), \\
& h_{1}^{(2)}(u)=\left(u+\sqrt{1-t^{2}}\right) g_{2}^{(2)}(u), \\
& h_{2}^{(2)}(u)=-\left(u+\sqrt{1-t^{2}}\right) g_{1}^{(2)}(u) .
\end{aligned}
$$

\subsection{The case $\boldsymbol{t} \leq-1$}

We can repeat the analysis, starting with (3.2) with $x<0$, but do not need new integral representations, algorithms or uniform asymptotic expansions for this case. For $U(a, x)$ we can use the second relation in (1.11), and for $V(a, x)$ the first relation.

When the parameter $a$ is large these relations have to be used with care, because gamma functions with large negative arguments occur. It is better to use the quantities $G_{j}, H_{j}$ introduced in $\$ 3.2 .1$. In the computer code these quantities will be given as output from the case $t>1$.

We have

$$
\begin{array}{r}
\begin{array}{r}
U(-a,-x)=\sqrt{\frac{2}{\pi}} a^{\frac{1}{4}} \gamma(a)\left\{e^{-2 a \xi}\left[\cos \pi a G_{2}+\sin \pi a G_{1}\right]\right. \\
\left.+e^{2 a \xi} \cos \pi a G_{3}\right\},
\end{array} \\
\begin{array}{r}
U^{\prime}(-a,-x)=-\sqrt{\frac{2}{\pi}} a^{\frac{3}{4}} \gamma(a)\left\{e^{-2 a \xi}\left[\cos \pi a H_{2}+\sin \pi a H_{1}\right]\right. \\
\left.+e^{2 a \xi} \cos \pi a H_{3}\right\},
\end{array} \\
\begin{array}{r}
V(-a,-x)=\sqrt{\frac{2}{\pi}} \frac{a^{\frac{1}{4}} \gamma(a)}{\Gamma\left(a+\frac{1}{2}\right)}\left\{e^{-2 a \xi}\left[\cos \pi a G_{1}-\sin \pi a G_{2}\right]\right. \\
\left.-e^{2 a \xi} \sin \pi a G_{3}\right\},
\end{array} \\
\begin{array}{r}
V^{\prime}(-a,-x)=-\sqrt{\frac{2}{\pi}} \frac{a^{\frac{3}{4}} \gamma(a)}{\Gamma\left(a+\frac{1}{2}\right)}\left\{e^{-2 a \xi}\left[\cos \pi a H_{1}-\sin \pi a H_{2}\right]\right. \\
\left.-e^{2 a \xi} \sin \pi a H_{3}\right\} .
\end{array}
\end{array}
$$




\section{The $W$-function}

In this section solutions of equation

$$
\frac{d^{2} y}{d x^{2}}+\left(\frac{1}{4} x^{2}-a\right) y=0
$$

are considered, again for real $a$ and $x$. For $a<0$ the solutions oscillate on the real $x$-axis; for $a>0$ there are turning points at $\pm 2 \sqrt{a}$, and the oscillations occur outside the interval $[-2 \sqrt{a}, 2 \sqrt{a}$. From quantum mechanics we know that (4.1) is the equation for propagation through a potential barrier.

\subsection{The standard solutions}

We consider solutions $W(a, x)$ and $W(a,-x)$; these form a numerically satisfactory pair for $-\infty<x<\infty$; see [5]. The function $W(a, x)$ has the initial values (see [1], p. 692)

$$
\begin{aligned}
& W(a, 0)=2^{-\frac{3}{4}}\left|\frac{\Gamma\left(\frac{1}{4}+\frac{1}{2} i a\right)}{\Gamma\left(\frac{3}{4}+\frac{1}{2} i a\right)}\right|^{\frac{1}{2}}, \\
& W^{\prime}(a, 0)=-2^{-\frac{1}{4}}\left|\frac{\Gamma\left(\frac{3}{4}+\frac{1}{2} i a\right)}{\Gamma\left(\frac{1}{4}+\frac{1}{2} i a\right)}\right|^{\frac{1}{2}} .
\end{aligned}
$$

The Wronskian of $W(a, x)$ and $W(a,-x)$ is

$$
\mathcal{W}[W(a, x), W(a,-x)]=1 .
$$

Power series expansions are

$$
W(a, x)=W(a, 0) w_{1}(a, x)+W^{\prime}(a, 0) w_{2}(a, x),
$$

where $w_{1}(a, x)$ and $w_{2}(a, x)$ are the even and odd solutions of (4.1). We have

$$
w_{1}(a, x)=\sum_{n=0}^{\infty} \alpha_{n}(a) \frac{x^{2 n}}{(2 n) !}, \quad w_{2}(a, x)=\sum_{n=0}^{\infty} \beta_{n}(a) \frac{x^{2 n+1}}{(2 n+1) !},
$$

where $\alpha_{n}(a), \beta_{n}(a)$ satisfy the recursion

$$
\begin{aligned}
& \alpha_{n+2}=a \alpha_{n+1}-\frac{1}{2}(n+1)(2 n+1) \alpha_{n}, \\
& \beta_{n+2}=a \beta_{n+1}-\frac{1}{2}(n+1)(2 n+3) \beta_{n}, \\
& \alpha_{0}(a)=1, \quad \alpha_{1}(a)=a, \quad \beta_{0}(a)=1, \quad \beta_{1}(a)=a .
\end{aligned}
$$


The relation with the function $U(a, x)$ reads

$$
\begin{aligned}
\frac{1}{\sqrt{k(a)}} W(a, x)+i \sqrt{k(a)} & W(a,-x) \\
= & \sqrt{2} e^{\frac{1}{4} \pi a+i \rho(a)} U\left(i a, x e^{-\pi i / 4}\right),
\end{aligned}
$$

which follows from using the initial values of the functions, but also from [1] [19.17.6 and 19.17.9]. The quantities $k(a)$ and $\rho(a)$ are given by

$$
k(a)=\sqrt{1+e^{2 \pi a}}-e^{\pi a}=\frac{1}{\sqrt{1+e^{2 \pi a}}+e^{\pi a}},
$$

and

$$
\rho(a)=\frac{1}{8} \pi+\frac{1}{2} \phi_{2}(a), \quad \phi_{2}(a)=\operatorname{ph} \Gamma\left(\frac{1}{2}+i a\right) ;
$$

the branch is defined by $\phi_{2}(0)=0$ and by continuity elsewhere.

Because we assume that $a$ and $x$, and hence $W(a, \pm x)$, are real, we have, using (4.7), that

$$
\begin{aligned}
& W(a, x)=\sqrt{2 k(a)} e^{\frac{1}{4} \pi a} \Re\left[e^{i \rho(a)} U\left(i a, x e^{-\pi i / 4}\right)\right], \\
& W(a,-x)=\sqrt{\frac{2}{k(a)}} e^{\frac{1}{4} \pi a} \Im\left[e^{i \rho(a)} U\left(i a, x e^{-\pi i / 4}\right)\right] .
\end{aligned}
$$

These relations are convenient for numerical computations because for $x \geq 0$ and $x \leq 0$ we can use the same $U$-function.

4.1.1 The function $\boldsymbol{\rho}(\boldsymbol{a})$ We give more details on the function $\rho$ defined in (4.9). For large values of $a$ it is convenient to use the representation

$$
\rho(a)=\frac{1}{8} \pi-\frac{1}{2} a+\frac{1}{4} a \ln a^{2}+\rho^{*}(a),
$$

where $\rho^{*}(a)=\mathcal{O}(1 / a)$ as $a \rightarrow \infty$. An asymptotic expansion follows from Binet's formula (see [9], p. 55, for an integrated version)

$$
\begin{aligned}
\ln \Gamma\left(z+\frac{1}{2}\right)= & z \ln \left(\frac{1}{2}+z\right)-\frac{1}{2}-z+\frac{1}{2} \ln (2 \pi) \\
& +\int_{0}^{\infty} \beta(t) e^{-\frac{1}{2} t} e^{-z t} d t
\end{aligned}
$$

where

$$
\begin{aligned}
\beta(t) e^{-\frac{1}{2} t} & =t^{-1}\left(\frac{1}{e^{t}-1}-\frac{1}{t}+\frac{1}{2}\right) e^{-\frac{1}{2} t} \\
& =\sum_{k=0}^{\infty} \frac{c_{k} t^{k}}{(k+2) !}, \quad|t|<2 \pi,
\end{aligned}
$$


with $c_{k}$ in terms of Bernoulli polynomials:

$$
c_{k}=B_{k+2}^{(1)}\left(-\frac{1}{2}\right)-(-1)^{k} \frac{k+3}{2^{k+2}}, \quad k=0,1,2, \ldots .
$$

This gives the asymptotic expansion

$$
\rho^{*}(a) \sim \frac{1}{4} a \ln \left(1+\frac{1}{4 a^{2}}\right)-\frac{1}{2 a} \sum_{k=0}^{\infty} \frac{d_{k}}{a^{2 k}},
$$

as $\pm a \rightarrow \infty$, where

$$
d_{k}=(-1)^{k} \frac{c_{2 k}}{(2 k+1)(2 k+2)}, \quad k=0,1,2, \ldots .
$$

The first few coefficients are

$d_{0}=\frac{1}{12}, d_{1}=-\frac{13}{720}, d_{2}=\frac{37}{20160}, d_{3}=-\frac{29}{26880}, d_{4}=-\frac{1129}{1520640}$.

\subsection{Integral representations for $\boldsymbol{a}<\mathbf{0}$}

For $W(-a, \pm x)$ we consider (2.1) for $U\left(-i a, x e^{-\pi i / 4}\right)$ (see (4.7) and (4.10)), that is,

$$
U\left(-i a, x e^{-\pi i / 4}\right)=\frac{e^{-\frac{1}{4} i x^{2}}}{i \sqrt{2 \pi}} \int_{\mathcal{C}} e^{-x e^{-\pi i / 4} s+\frac{1}{2} s^{2}} s^{i a} \frac{d s}{\sqrt{s}},
$$

where $\mathcal{C}$ is a vertical line on which $\Re s>0$. On $\mathcal{C}$ we have $-\frac{1}{2} \pi<$ ph $s<\frac{1}{2} \pi$, and the many-valued function $s^{i a-\frac{1}{2}}$ assumes its principal value. The transformations

$$
x=2 t \sqrt{a}, \quad s=\sqrt{a} w
$$

give

$$
U\left(-i a, x e^{-\pi i / 4}\right)=\frac{e^{-\frac{1}{4} i x^{2}} a^{\frac{1}{4}+\frac{1}{2} a i}}{i \sqrt{2 \pi}} \int_{\mathcal{C}} e^{a \phi(w)} \frac{d w}{\sqrt{w}}
$$

where

$$
\phi(w)=\frac{1}{2} w^{2}-2 t e^{-\pi i / 4} w+i \ln w .
$$

The saddle points follow from solving

$$
\phi^{\prime}(w)=\frac{w^{2}-2 t e^{-\pi i / 4} w+i}{w}=0,
$$


giving saddle points

$$
w_{ \pm}=u_{ \pm}+i v_{ \pm}=e^{-\pi i / 4}\left(t \pm \sqrt{t^{2}+1}\right), \quad v_{ \pm}=-u_{ \pm} .
$$

The relevant saddle point is $w_{+}$. We have

$$
\phi\left(w_{+}\right)=\frac{1}{4} \pi+i\left(t^{2}+2 \widetilde{\xi}-\frac{1}{2}\right),
$$

where $\tilde{\xi}$ is given in (2.12). The path of steepest descent through $w_{+}$ is for $|\theta|<\frac{1}{2} \pi$ defined by

$$
\frac{1}{2} r^{2} \sin 2 \theta-2 t r \sin \left(\theta-\frac{1}{4} \pi\right)+\ln r=\Im \phi\left(w_{+}\right)=t^{2}+2 \widetilde{\xi}-\frac{1}{2},
$$

where $w=r e^{i \theta}$. In rectangular coordinates $w=u+i v$ this equation reads

$$
u v+\sqrt{2} t(u-v)+\frac{1}{2} \ln \left(u^{2}+v^{2}\right)=t^{2}+2 \widetilde{\xi}-\frac{1}{2} .
$$

We can solve equation (4.25) for $\sin \left(\theta-\frac{1}{4} \pi\right.$ ) (it is a quadratic equation for this quantity), giving $\theta$ as function of $r$. This makes it possible to integrate (4.20) with respect to $r$, but this introduces singularities in the integral where $r$ attains its minimal value, although the path itself is smooth.

Integrating with respect to $\theta$ or $v$ is a better option. We can numerically determine the path in an algorithm, but this is not a very efficient method. Instead, we replace the steepest descent path defined in (4.26) by a path $u(v)$ such that

1. $u(v)$ is smooth for all $v \in \mathbb{R}$;

2. $u(v)$ passes through the saddle point: $u\left(v_{+}\right)=u_{+}$;

3. $d u / d v$ at $v_{+}$has the same value as $d u / d v$ for the steepest descent contour at $w_{+}$;

4. the path runs into the valleys of $e^{\phi(w)}$ at $\pm i \infty$.

¿From (4.26) we can show that $d u / d v=0$ at the saddle point $w_{+}$. Hence, a simple path $\mathcal{C}$ that fulfills the four conditions is the vertical line $u=u_{+}$. Introducing $q=v-v_{+}$, using $w=w_{+}+i q$ and (4.24) we obtain for (4.20) the representation

$$
\begin{aligned}
U\left(-i a, x e^{-\pi i / 4}\right)=\frac{e^{\frac{1}{4} \pi a-\frac{1}{2} i a} a^{\frac{1}{4}+\frac{1}{2} a i}}{\sqrt{2 \pi w_{+}}} e^{2 i a \tilde{\xi}} \\
\times \int_{-\infty}^{\infty} e^{-a \psi(q)} g(q) d q .
\end{aligned}
$$


The function $\psi(q)$ is given by

$$
\psi(q)=\phi\left(w_{+}\right)-\phi(w), \quad g(q)=\frac{1}{\sqrt{1+i q / w_{+}}} .
$$

For small values of $q$ we have

$$
\begin{aligned}
& \psi(q)=\frac{1+2 u_{+}^{2}}{4 u_{+}^{2}} q^{2}+\frac{1-i}{12 u_{+}^{3}} q^{3}+\mathcal{O}\left(q^{4}\right), \\
& u_{+}=\frac{1}{\sqrt{2}}\left(t+\sqrt{t^{2}+1}\right) .
\end{aligned}
$$

We conclude that $W(-a, \pm x)$ (see (4.7) - (4.11)) are given by

$$
\begin{gathered}
W(-a, x)=\frac{a^{\frac{1}{4}} \sqrt{k(-a)}}{\sqrt{\pi\left|w_{+}\right|}} \Re\left[e^{i \chi} \int_{-\infty}^{\infty} e^{-a \psi(q)} g(q) d q\right], \\
W(-a,-x)=\frac{a^{\frac{1}{4}}}{\sqrt{\pi\left|w_{+}\right| k(-a)}} \Im\left[e^{i \chi} \int_{-\infty}^{\infty} e^{-a \psi(q)} g(q) d q\right],
\end{gathered}
$$

where

$$
\chi=\rho^{*}(-a)+\frac{1}{4} \pi+2 a \widetilde{\xi} .
$$

For the derivatives we find, starting with (4.18),

$$
\begin{aligned}
e^{-\pi i / 4} U^{\prime}\left(-i a, x e^{-\pi i / 4}\right)=i \frac{e^{\frac{1}{4} \pi a-\frac{1}{2} i a} a^{\frac{3}{4}+\frac{1}{2} a i}}{\sqrt{2 \pi w_{+}}} e^{2 i a \widetilde{\xi}} \\
\quad \times \int_{-\infty}^{\infty} e^{-a \psi(q)} h(q) d q,
\end{aligned}
$$

where

$$
h(q)=\left(\sqrt{t^{2}+1}-e^{-\pi i / 4} q\right) g(q) .
$$

It follows from (4.10) that $W^{\prime}(-a, \pm x)$ are given by

$$
\begin{aligned}
& W^{\prime}(-a, x)=\frac{a^{\frac{3}{4}} \sqrt{k(-a)}}{\sqrt{\pi\left|w_{+}\right|}} \Re\left[i e^{i \chi} \int_{-\infty}^{\infty} e^{-a \psi(q)} h(q) d q\right], \\
& W^{\prime}(-a,-x)=-\frac{a^{\frac{3}{4}}}{\sqrt{\pi\left|w_{+}\right| k(-a)}} \Im\left[i e^{i \chi} \int_{-\infty}^{\infty} e^{-a \psi(q)} h(q) d q\right] .
\end{aligned}
$$

For large values of $a$ and/or $t$ the oscillatory behaviour of $W(-a, \pm x)$ and $W^{\prime}(-a, \pm x)$ is mainly described by the exponential factor $e^{2 i a \widetilde{\xi}}$ contained in $e^{i \chi}$. The other elements of these formulas are slowly varying. 
Asymptotic expansions follow from [10]. Formula (2.29) of that paper gives, with $\mu=\sqrt{2 a} e^{-\pi i / 4}$,

$$
W(-a, x) \sim \frac{\sqrt{k(-a)}}{a^{\frac{1}{4}}\left(t^{2}+1\right)^{\frac{1}{4}}} \Re\left[e^{i \chi} \sum_{s=0}^{\infty} \frac{(-i)^{s} \phi_{s}(\widetilde{\tau})}{(2 a)^{s}}\right],
$$

where $\phi_{s}$ are polynomials given in (2.11) and $\widetilde{\tau}$ in (2.32) of [10].

Formula (2.33) of [10] gives

$$
W^{\prime}(-a, x) \sim \sqrt{k(-a)} a^{\frac{1}{4}}\left(t^{2}+1\right)^{\frac{1}{4}} \Re\left[i e^{i \chi} \sum_{s=0}^{\infty} \frac{(-i)^{s} \psi_{s}(\widetilde{\tau})}{(2 a)^{s}}\right],
$$

where $\psi_{s}$ are polynomials given in (2.16) of [10].

For $W(-a,-x)$ and its derivative we have

$$
W(-a,-x) \sim \frac{\sqrt{k(-a)}}{a^{\frac{1}{4}}\left(t^{2}+1\right)^{\frac{1}{4}}} \Im\left[e^{i \chi} \sum_{s=0}^{\infty} \frac{(-i)^{s} \phi_{s}(\widetilde{\tau})}{(2 a)^{s}}\right],
$$

and

$W^{\prime}(-a,-x) \sim-\sqrt{k(-a)} a^{\frac{1}{4}}\left(t^{2}+1\right)^{\frac{1}{4}} \Im\left[i e^{i \chi} \sum_{s=0}^{\infty} \frac{(-i)^{s} \psi_{s}(\widetilde{\tau})}{(2 a)^{s}}\right]$,

The asymptotic expansions in (4.37) - (4.40) hold when $a \rightarrow \infty$, uniformly with respect to $t \geq-t_{0}$, but also for $t \rightarrow \infty$, uniformly with respect to $a \geq a_{0}$, where $a_{0}$ and $t_{0}$ are fixed positive numbers.

\subsection{Integral representations for $\boldsymbol{a}>\mathbf{0}$}

Because of the turning points we consider three cases. We write $x=$ $2 t \sqrt{a}$. We use the $U$-function in (4.7), and write (2.1) in the form

$$
U\left(i a, x e^{-\pi i / 4}\right)=\frac{e^{-\frac{1}{4} i x^{2}}}{i \sqrt{2 \pi}} \int_{\mathcal{C}} e^{-x e^{-\pi i / 4} s+\frac{1}{2} s^{2}} s^{-i a} \frac{d s}{\sqrt{s}},
$$

with conditions as in (4.18). The transformation $s=\sqrt{a} w$ gives

$$
U\left(i a, x e^{-\pi i / 4}\right)=\frac{e^{-\frac{1}{4} i x^{2}} a^{\frac{1}{4}-\frac{1}{2} a i}}{i \sqrt{2 \pi}} \int_{\mathcal{C}} e^{a \phi(w)} \frac{d w}{\sqrt{w}},
$$

where

$$
\phi(w)=\frac{1}{2} w^{2}-2 t e^{-\pi i / 4} w-i \ln w
$$


4.3.1 The case $t \geq 1$ The saddle points are now

$$
w_{ \pm}=u_{ \pm}+i v_{ \pm}=e^{-\pi i / 4}\left(t \pm \sqrt{t^{2}-1}\right), \quad v_{ \pm}=-u_{ \pm} .
$$

The relevant saddle point is $w_{+}$, and for numerical integration a convenient choice of $\mathcal{C}$ is the vertical line through $w_{+}$.

Using

$$
-\frac{1}{4} i x^{2}+a \phi\left(w_{+}\right)=2 i a \xi+\frac{1}{2} i a-\frac{1}{4} \pi a,
$$

where $\xi$ is given in (3.38), and writing in (4.42) $w=w_{+}+i q$, we obtain the analogue of (4.27)

$$
U\left(i a, x e^{-\pi i / 4}\right)=\frac{e^{-\frac{1}{4} \pi a+\frac{1}{2} i a} a^{\frac{1}{4}-\frac{1}{2} a i}}{\sqrt{2 \pi w_{+}}} e^{2 i a \xi} \int_{-\infty}^{\infty} e^{-a \psi(q)} g(q) d q
$$

where

$$
\psi(q)=\phi\left(w_{+}\right)-\phi(w), \quad g(q)=\frac{1}{\sqrt{1+i q / w_{+}}} .
$$

It follows that

$$
W(a, x)=\frac{\sqrt{k(a)} a^{\frac{1}{4}}}{\sqrt{\pi\left|w_{+}\right|}} \Re[G(a, x)]
$$

and

$$
W(a,-x)=\frac{\sqrt{k(a)} a^{\frac{1}{4}}}{\sqrt{\pi\left|w_{+}\right|}} \Im[G(a, x)]
$$

where

$$
G(a, x)=e^{i\left[\rho^{*}(a)+\frac{1}{4} \pi+2 i a \xi\right]} \int_{-\infty}^{\infty} e^{-a \psi(q)} g(q) d q
$$

For the derivative we find, as in (4.33),

$$
\begin{aligned}
U^{\prime}\left(i a, x e^{-\pi i / 4}\right)=-\frac{e^{-\frac{1}{4} \pi a+\frac{1}{2} i a} a^{\frac{3}{4}-\frac{1}{2} a i}}{\sqrt{2 \pi w_{+}}} e^{2 i a \xi} \\
\quad \times \int_{-\infty}^{\infty} e^{-a \psi(q)} h(q) d q,
\end{aligned}
$$

where

$$
h(q)=\left(e^{-\pi i / 4} \sqrt{t^{2}-1}+i q\right) g(q) .
$$

It follows that

$$
W^{\prime}(a, x)=-\frac{\sqrt{k(a)} a^{\frac{3}{4}}}{\sqrt{\pi}\left|w_{+}\right|} \Re[H(a, x)]
$$


and

$$
W^{\prime}(a,-x)=\frac{a^{\frac{3}{4}}}{\sqrt{\pi k(a)}\left|w_{+}\right|} \Im[H(a, x)]
$$

where

$$
H(a, x)=e^{i\left[\rho^{*}(a)+\frac{1}{4} \pi+2 i a \xi\right]} \int_{-\infty}^{\infty} e^{-a \psi(q)} h(q) d q
$$

Asymptotic expansions follow from (2.9) of [10]. By changing $\mu \rightarrow$ $\mu e^{-\pi i / 4}$ in that formula we obtain

$$
W(a, x) \sim \frac{\sqrt{k(a)}}{a^{\frac{1}{4}}\left(t^{2}-1\right)^{\frac{1}{4}}} \Re\left[e^{i\left[\rho^{*}(a)+\frac{1}{4} \pi+2 a \xi\right]} \sum_{s=0}^{\infty} \frac{i^{s} \phi_{s}(\tau)}{(2 a)^{s}}\right],
$$

where $\phi_{s}(\tau)$ are the same polynomials as in (4.37), and

$$
\tau=\frac{1}{2}\left(\frac{t}{\sqrt{t^{2}-1}}-1\right)
$$

Formula (2.18) of [10] gives

$$
\begin{aligned}
& W^{\prime}(a, x) \sim-\sqrt{k(a)} a^{\frac{1}{4}}\left(t^{2}-1\right)^{\frac{1}{4}} \\
& \times \Re\left[e^{i\left[\rho^{*}(a)-\frac{1}{4} \pi+2 a \xi\right]} \sum_{s=0}^{\infty} \frac{i^{s} \psi_{s}(\tau)}{(2 a)^{s}}\right],
\end{aligned}
$$

where $\psi_{s}(\tau)$ are the same as in (4.38).

The asymptotic expansions in (4.56) and (4.58) hold when $a \rightarrow \infty$, uniformly with respect to $t \geq 1+t_{0}$, but also for $t \rightarrow \infty$, uniformly with respect to $a \geq a_{0}$, where $a_{0}$ and $t_{0}$ are positive numbers.

4.3.2 The case $-1 \leq t \leq 1 \quad$ We use (4.41), (4.42) and (4.43) with saddle points

$$
w_{ \pm}=e^{-\pi i / 4}\left(t \pm i \sqrt{1-t^{2}}\right)=e^{-\pi i / 4 \pm i \theta}, \quad t=\cos \theta
$$

which are located on the unit circle. We have

$$
\phi\left(w_{ \pm}\right)= \pm 2 \eta-\frac{1}{4} \pi+i\left(\frac{1}{2}+t^{2}\right)
$$

where $\eta=\frac{1}{2}(\theta-\sin \theta \cos \theta)$ is also used in $\S 3.1$ and defined in (3.9). We see that the imaginary parts of $\phi\left(w_{ \pm}\right)$are equal. As a consequence, the steepest descent path may go (and in fact in the present case does go) through both saddle points.

In Figure 3 we have shown the paths for three values of $t$. The contours run from $-i \infty$ to $w_{-}$, then along the arc to $w_{+}$(in the 
direction of the arrows), and from $w_{+}$to $+i \infty$. Through each saddle point the local contours of steepest ascent and steepest ascent are shown. The complete contours include steepest descent parts and steepest ascent parts.

From (4.60) we see that $w_{+}$is dominant for $0 \leq t<1$ ( $\eta$ is positive for these values of $t)$. Another point of interest is that the oscillatory factor $e^{-\frac{1}{4} i x^{2}}=e^{-i a t^{2}}$ in (4.42) is nullified when we put $\phi\left(w_{-}\right)$or $\phi\left(w_{+}\right)$in front of the integral, because $\Im \phi\left(w_{ \pm}\right)=\frac{1}{2}+t^{2}$. This explains that the function $W(a, x)$ does not oscillate if $t \in[-1,1]$.
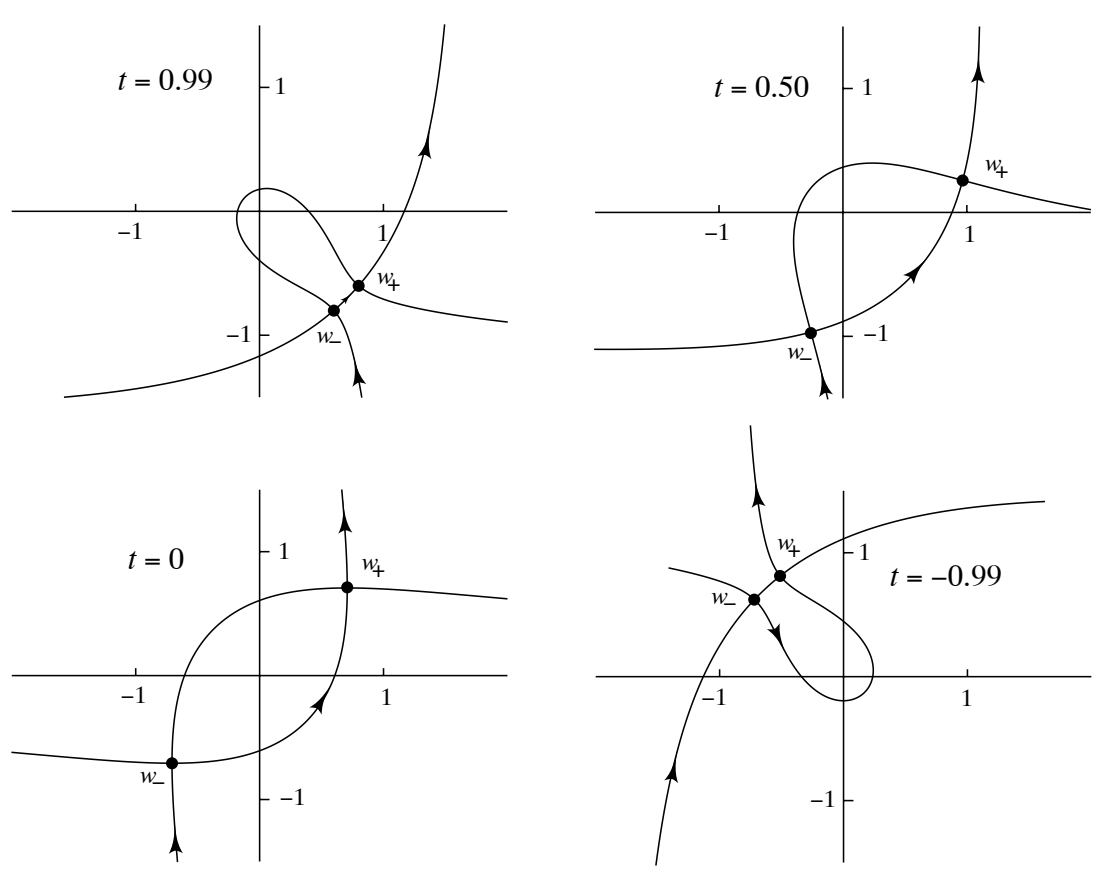

Figure 3. Steepest descent contour for the integral in (4.61) for several $t$-values. The contours run from $-i \infty$ to $w_{-}$, then along the arc to $w_{+}$(in the direction of the arrow), and from $w_{+}$ to $+i \infty$. Through each saddle point the local contours of steepest ascent and steepest ascent are shown. The complete contours include steepest descent parts and steepest ascent parts. 
When we write $w=u+i v$ and integrate in (4.42) with respect to $v$ we obtain

$$
\begin{aligned}
& U\left(i a, x e^{-\pi i / 4}\right)=\frac{a^{\frac{1}{4}-\frac{1}{2} a i} e^{a\left[\frac{1}{2} i-\frac{1}{4} \pi+2 \eta\right]}}{\sqrt{2 \pi}} \times \\
& {\left[\int_{v_{-}}^{\infty} e^{-a \psi_{1}(v)} f_{1}(v) d v+e^{-4 a \eta} \int_{-\infty}^{v_{-}} e^{-a \psi_{2}(v)} f_{2}(v) d v\right],}
\end{aligned}
$$

where

$$
\psi_{1}(v)=\phi\left(w_{+}\right)-\phi(w), \quad \psi_{2}(v)=\phi\left(w_{-}\right)-\phi(w),
$$

and

$$
f_{1}(v)=\frac{1}{\sqrt{w}}\left(1-i \frac{d u_{1}}{d v}\right), \quad f_{2}(v)=\frac{1}{\sqrt{w}}\left(1-i \frac{d u_{2}}{d v}\right) .
$$

We may assume different relations between $u$ and $v$ in both integrals; this explains $u_{1}$ and $u_{2}$, which are functions of $v$.

It follows from (4.10) that

$$
\begin{aligned}
& W(a, x)=\frac{\sqrt{k(a)} a^{\frac{1}{4}} e^{2 a \eta}}{\sqrt{\pi}} \Re\left[e^{i\left[\rho^{*}(a)+\frac{1}{8} \pi\right]} K(a, x)\right], \\
& W(a,-x)=\frac{a^{\frac{1}{4}} e^{2 a \eta}}{\sqrt{\pi k(a)}} \Im\left[e^{i\left[\rho^{*}(a)+\frac{1}{8} \pi\right]} K(a, x)\right],
\end{aligned}
$$

where $K(a, x)$ denotes the sum of the integrals between the square brackets in (4.61).

For the derivatives we find, starting with (4.42),

$$
U^{\prime}\left(i a, x e^{-\pi i / 4}\right)=\frac{e^{-\frac{1}{4} i x^{2}} a^{\frac{3}{4}-\frac{1}{2} a i}}{\sqrt{2 \pi}} \int_{\mathcal{C}} e^{a \phi(w)}\left(t e^{-\pi i / 4}-w\right) \frac{d w}{\sqrt{w}},
$$

and using (4.10),

$$
\begin{aligned}
& W^{\prime}(a, x)=\frac{\sqrt{k(a)} a^{\frac{3}{4}} e^{2 a \eta}}{\sqrt{\pi}} \Re\left[e^{i\left[\rho^{*}(a)-\frac{1}{8} \pi\right]} K_{d}(a, x)\right], \\
& W^{\prime}(a,-x)=-\frac{a^{\frac{3}{4}} e^{2 a \eta}}{\sqrt{\pi k(a)}} \Im\left[e^{i\left[\rho^{*}(a)-\frac{1}{8} \pi\right]} K_{d}(a, x)\right],
\end{aligned}
$$

where $K_{d}(a, x)$ denotes the sum of the integrals between the square brackets in (4.61) with $f_{i}(v)$ replaced with $g_{i}(v)=\left(t e^{-\frac{1}{4} \pi i}-w\right) f_{i}(v)$. 
The asymptotic expansions follows from (2.29) and (2.33) of [10]. We change $t \rightarrow-i t$ and $\mu \rightarrow \mu e^{\frac{1}{4} \pi i}$ in these formulae and obtain

$$
\begin{gathered}
U\left(i a, x e^{-\pi i / 4}\right) \sim \frac{a^{-\frac{1}{2} i a-\frac{1}{4}} e^{\frac{1}{2} i a-\frac{1}{8} \pi i-\frac{1}{4} \pi a+2 a \eta}}{\sqrt{2}\left(1-t^{2}\right)^{\frac{1}{4}}} \sum_{s=0}^{\infty} i^{s} \frac{\phi_{s}\left(\tau^{*}\right)}{(2 a)^{s}}, \\
U^{\prime}\left(i a, x e^{-\pi i / 4}\right) \sim-\frac{a^{-\frac{1}{2} i a+\frac{1}{4}} e^{\frac{1}{2} i a+\frac{1}{8} \pi i-\frac{1}{4} \pi a+2 a \eta}\left(1-t^{2}\right)^{\frac{1}{4}}}{\sqrt{2}} \\
\times \sum_{s=0}^{\infty} i^{s} \frac{\psi_{s}\left(\tau^{*}\right)}{(2 a)^{s}}
\end{gathered}
$$

where $\eta$ is defined in (3.9) and $\phi_{s}\left(\tau^{*}\right)$ are the same as in (3.35).

It follows from (4.10) that

$$
\begin{gathered}
W(a, x) \sim \frac{\sqrt{k(a)}}{a^{\frac{1}{4}}\left(1-t^{2}\right)^{\frac{1}{4}}} e^{2 a \eta} \Re\left[e^{i \rho^{*}(a)} \sum_{s=0}^{\infty} \frac{i^{s} \phi_{s}\left(\tau^{*}\right)}{(2 a)^{s}}\right], \\
W^{\prime}(a, x) \sim-\sqrt{k(a)} a^{\frac{1}{4}}\left(1-t^{2}\right)^{\frac{1}{4}} e^{2 a \eta} \\
\times \Re\left[e^{i\left[\rho^{*}(a)+\frac{1}{4} \pi\right]} \sum_{s=0}^{\infty} \frac{i^{s} \psi_{s}\left(\tau^{*}\right)}{(2 a)^{s}}\right] .
\end{gathered}
$$

The asymptotic expansions in (4.68) hold when $a \rightarrow \infty$, uniformly with respect to $t \in[-1+\delta, 1-\delta]$, where $\delta$ is a fixed positive number.

4.3.3 Unstable representations For large values of a $\eta$ the representations for $W(a,-x)$ and $W^{\prime}(a,-x)$ in (4.64) and (4.66) are unstable. To see this, observe that (4.68) can be used for $t \in[-1+\delta, 1-\delta]$. The dominant behaviour comes from $\sqrt{k(a)} e^{2 a \eta}$. Since (see (4.8)) $k(a) \sim \frac{1}{2} e^{-\pi a}$, the dominant behaviour comes from $e^{a \chi}$, where $\chi=$ $\arcsin t-t \sqrt{1-t^{2}}$, an odd function that is positive on $(0,1]$. This dominant behaviour does not appear in the representations for $W(a,-x)$ and $W^{\prime}(a,-x)$ in (4.64) and (4.66). There we see the dominant parts $e^{2 a \eta} / \sqrt{k(a)}$; in $\eta$ we use positive $t$ when $x$ is positive. It follows that the imaginary parts in the right-hand sides of (4.64) and (4.66) have to be very small when $a \eta$ is large. In fact, the first integral in (4.61) should be of order $e^{-4 a \eta}$ in that case, which is not apparent from this representation.

A possible solution to this problem is using the representations for $W(a, x)$ and $W^{\prime}(a, x)$ in (4.64) and (4.66) for $t \in[-1,0]$. However, when $t \downarrow-1$ the phase of $w_{+}$becomes $3 \pi / 4$ and that of $w_{-}$becomes $-5 \pi / 4$, which is outside the standard interval $(-\pi, \pi]$ of the phase of $w$ in (4.42); that is, $w$ is outside the standard Riemann sheet. In 
Figure 3 the path for the case $t=-0.99$ is shown. The technical details will be worked out when writing the numerical algorithms.

\section{Concluding remarks}

In a future paper we will discuss the numerical aspects and describe computer algorithms based on the integral representations given in this paper. Several quantities have to be calculated with great care. For example, straightforward use of $\psi(\theta)$ defined in (2.10) when $\theta$ is small, that is, at the saddle point, will give cancellation of leading digits. Also, to represent the functions for a large range of the parameters scaling is needed.

When implementing the representations we will decide if the steepest descent paths will be used or approximations of these paths, as we suggested for the $W$-function in $\S 4.2$. For example, integrating in (2.3) along the vertical line through the saddle point $w_{0}$ gives a simpler representation than (2.9). However, the integral along the vertical line has a non-real phase function. Another approximation of a steepest descent contour is given in (3.13). We will investigate efficiency aspects in combination with programming aspects in deciding which representation in these examples should be used.

This also holds for the quite complicated steepest descent paths in $\S 4.3 .2$. We have not indicated in (4.61) the relation between $v$ and $u$ on the different parts of the path. This will be done during the implementation of the algorithms.

\section{Acknowledgments}

The authors thank the referees for their careful reading of the manuscript and their valuable comments. A. Gil acknowledges financial support from Ministerio de Ciencia y Tecnología (BFM2001-3878C02-01).

\section{References}

1. M. Abramowitz and I.A. Stegun (1964), Handbook of mathematical functions with formulas, graphs and mathematical tables, Nat. Bur. Standards Appl. Series, 55, U.S. Government Printing Office, Washington, D.C. (paperback edition published by Dover, New York).

2. Gil A., J. Segura, N.M. Temme (2001), On non-oscillating integrals for computing inhomogeneous Airy functions. Math. Comput., 70, 1183-1194. 
3. D.W. Lozier and F.W.J. Olver (1994), Numerical evaluation of special functions, in W. Gautschi (ed.), Mathematics of computation 1943-1993: A halfcentury of computational mathematics, PSAM, AMS, Providence.

4. J.C.P. Miller (1952), On the choice of standard solutions to Weber's equation, Proc. Cambridge Philos. Soc., 48, 428-435.

5. J.C.P. Miller (1955), Tables of Weber parabolic cylinder functions. H.M. Stationary Office, London.

6. F.W.J. Olver (1959), Uniform asymptotic expansions for Weber parabolic cylinder functions of large order, J. Research NBS, 63B, 131-169.

7. F.W.J. Olver (1974 \& 1997), Asymptotics and Special Functions. Academic Press, New York. Reprinted in 1997 by A.K. Peters,

8. N.M. Temme (1994), Steepest descent paths for integrals defining the modified Bessel functions of imaginary order. Methods Appl. Anal. 1, 14-24.

9. N.M. Temme (1996), Special functions: An introduction to the classical functions of mathematical physics, John Wiley and Sons, New York.

10. N.M. Temme (2000), Numerical and asymptotic aspects of parabolic cylinder functions, J. Comp. Appl. Math., 121, 221-246.

11. R. Wong (1989), Asymptotic approximations of integrals, Academic Press, New York. Reprinted in 2001 by SIAM. 